\title{
Diversité des insectes floricoles et son impact sur les rendements fruitiers et grainiers de Glycine max (Fabaceae) à Yassa (Douala, Cameroun)
}

\author{
TAIMANGA $^{1,2^{*}}$ et Fernand-Nestor TCHUENGUEM FOHOUO ${ }^{1}$ \\ ${ }^{1}$ Université de Ngaoundéré, Faculté des Sciences, Laboratoire de Zoologie Appliquée, \\ B.P.: 454 Ngaoundéré, Cameroun. \\ ${ }^{2}$ Université de Douala, Institut des Sciences Halieutiques, \\ Laboratoire des Ressources Halieutiques, B.P.: 7236 Douala, Cameroun. \\ *Auteur correspondant, E-mail: taimanga2003@yahoo.fr, Tel: (+237) 675208331
}

\section{RESUME}

Afin d'évaluer l'impact des insectes anthophiles sur les rendements de Glycine max (Fabaceae), des observations ont été faites sur les fleurs de cette Fabaceae de juin à juillet 2013 puis de mai à juin 2014 à Yassa. Deux traitements ont été déterminés à partir du marquage de six parcelles différenciées par la présence ou l'absence de protection des plantes vis-à-vis des insectes. L'activité de butinage et de pollinisation des insectes a été étudiée. Le taux de fructification, le pourcentage de graines normales et le nombre moyen de graines par gousse ont été évalués. Les résultats montrent que 13 et 14 espèces d'insectes ont visité les fleurs du soja respectivement en 2013 et 2014. Sur les fleurs de G. max, les insectes récoltaient intensément et régulièrement le pollen et le nectar. Lasioglossum sp. et Leuconomia granulata sont respectivement les espèces les plus fréquemment observées en 2013 et 2014. Par le biais de leur activité pollinisatrice, les insectes anthophiles ont augmenté le taux de fructification de $37,92 \%$ et $41,91 \%$, le pourcentage de graines normales de $20,86 \%$ et $40,57 \%$, le nombre moyen de graines par gousse de $12,76 \%$ et $12,60 \%$ respectivement en 2013 et 2014 . La protection des nids des Halactidés dans les plantations de G. max est recommandée pour accroitre la production des gousses et des graines.

(C) 2018 International Formulae Group. All rights reserved.

Mots clés: Insectes anthophiles, Glycine max, butinage, pollen, nectar, rendements.

\section{Diversity of flowering insects and its impact on Glycine max (Fabaceae) fruit and seed yields at Yassa (Douala, Cameroon)}

\begin{abstract}
To estimate the impact of anthophilous insects on Glycine max (Fabaceae) yields, the flowers of this Fabaceae were observed from June to July 2013 and from May to June 2014 at Yassa. Two treatments, differentiated according to plants were protected from insects activities or not. The pollination and behavior of foragers were studied. The fructification rate, the number of seeds per pod and the percentage of normal seeds were evaluated. In 2013 and 2014, 13 and 14 species of insects were inventoried on the Glycine max flowers respectively. This insect intensely harvested pollen and nectar. In 2013 and 2014 Lasioglossum sp. and Leuconomia granulata were the most frequent insects respectively. Via their pollination behavior anthophilous insects have increased the fructification rate by $37.92 \%$ and $41.91 \%$, the number of seeds per pod by $12.76 \%$
\end{abstract}


and $12.60 \%$ and the percentage of normal seeds by $20.86 \%$ and $40.57 \%$ respectively in 2013 and 2014 . The protection of Halictidae nests close to the soybean field is recommended to improve its fruit and seed yields. (C) 2018 International Formulae Group. All rights reserved.

Keywords: anthophilous insects, Glycine max, pollen, nectar, yields.

\section{INTRODUCTION}

Les insectes floricoles ont une grande importance écologique et économique, du fait qu'ils participent à la stabilisation de la biodiversité végétale par leur contribution à la production de graines et de fruits (Morisson et al., 2000; Tchuenguem et al., 2002). Cependant, l'exploitation des insectes pollinisateurs figure rarement dans les programmes agricoles de plusieurs pays africains. En effet, la majorité des agriculteurs de ce continent ne savent pas qu'en absence des insectes pollinisateurs ou en présence d'un nombre insuffisant de ces arthropodes au cours de la floraison, les rendements peuvent être fortement réduits ou nuls (Dounia, 2015; Tchuenguem, 2005; Vaughton et al., 2010). Pour eux, l'obtention des rendements élevés passe principalement par l'utilisation des engrais et des pesticides (DSCE, 2010; DSCE, 2009).

$\mathrm{Au}$ Cameroun, la connaissance des relations entre les espèces végétales et leurs insectes floricoles est en forte progression grâce notamment aux recherches menées depuis plus de 27 ans dans les Régions du Centre (Tchuenguem et al., 2001, 2002; Kengue et al., 2002; Pando et al., 2014), de l'Adamaoua (Tchuenguem, 2005; Djonwangwe et al., 2011; Noubissié et al., 2012; Kingha et al., 2014), de l'Extrême-Nord (Tchuenguem et Dounia, 2014) et du NordOuest (Otiobo et al., 2015). Malgré ces importants travaux, les interactions entre plusieurs plantes cultivées dans diverses régions du Cameroun et les insectes anthophiles sont encore inconnues. En outre, dans ce pays, la population à nourrir va sans cesse croissante et les productions agricoles demeurent faibles (DSCE, 2009). Il s'avère nécessaire de prendre en compte le facteur « insectes pollinisateurs » dans les programmes d'amélioration des rendements des cultures, afin d'obtenir les rendements plus élevés par unité de surface cultivée (Tchuenguem, 2005).

Glycine max fut introduit en Afrique au début du $19^{\text {ème }}$ siècle (Raemaekers, 2001). Actuellement, l'Egypte et le Nigeria sont les plus grands producteurs africains du soja ; tandis qu'au Cameroun, sa production reste encore trop faible (Raemaekers, 2001). Plante herbacée annuelle pouvant atteindre $110 \mathrm{~cm}$ de hauteur à maturité, les feuilles alternes sont composées et généralement formées de trois folioles (Gallais et al., 2009). L'inflorescence est une grappe portant 5 à 35 fleurs de couleur blanche, jaune ou rouge ; le nectar et le pollen produit par ces fleurs attirent les insectes (Milfont et al., 2013). Les graines sont largement utilisées dans l'alimentation humaine (Ngamo et al., 2016; Tien et al., 2002). Malgré son importance alimentaire et économique, il n'existe pas, à notre connaissance, de données publiées sur les relations entre $G$. $\max$ et ses insectes anthophiles dans la région du Littoral au Cameroun. Les seuls travaux effectués sur les relations $G$. max-insectes au Cameroun sont ceux de Kengni et al. (2015) dans la ville de Ngaoundéré (région de l'Adamaoua) et Tchuenguem et Dounia (2014) dans la ville de Maroua (région de l'Extrême Nord). Il ressort respectivement de ces travaux, à Ngaoundéré et Maroua, que: 7 et 28 espèces d'insectes visitent les fleurs du soja pour des raisons alimentaires. Apis mellifera adansonii est l'espèce la plus abondante $(76,15 \%$ et $23,18 \%$ des visites) et récolte exclusivement le nectar; les insectes, dans l'ensemble, sont actifs 
durant toute la période de floraison; leurs visites sont plus nombreuses dans la matinée; le comportement de butinage varie avec les insectes; via la pollinisation, les insectes anthophiles augmentent le nombre de gousses par plante de $35,85 \%$ et $31,29 \%$ et accroissent le nombre de grains par gousse de $73,09 \%$ et $22,85 \%$. Or l'activité des insectes sur les fleurs d'une plante peut varier dans l'espace et le temps.

Pour une meilleure gestion des relations sus évoquées, il est nécessaire de mener d'autres recherches sur les rapports entre G. $\max$ et ses insectes floricoles dans la région du Littoral, pour leur gestion optimale.

Les objectifs spécifiques incluent: l'inventaire des insectes anthophiles de $G$. max; l'étude de l'activité des insectes sur ses fleurs; l'estimation de la valeur apicole de cette Fabaceae; l'évaluation de l'impact des insectes floricoles sur la pollinisation et les rendements en gousses et en graines de cette plante.

\section{MATERIEL ET METHODES \\ Site, champ expérimental et matériel \\ biologique}

Les travaux ont été effectués de juin à juillet 2013 et de mai à juin 2014, à Yassa (Douala) dans la région du Littoral au Cameroun. Cette région appartient à la zone écologique dite forestière mono-modale; le climat est du type camerounien, caractérisé par une saison pluvieuse (avril à minovembre) et une saison sèche (mi-novembre à mars); la température moyenne est de 26 ${ }^{\circ} \mathrm{C}$; l'hygrométrie est de $80 \%$ en saison sèche et de $99 \%$ en saison des pluies (Gilles, 2000).

Le champ expérimental était une aire de $200 \mathrm{~m}^{2}$ centré sur un point de latitude: $4^{\circ} 00.469^{\prime} \mathrm{N}$, de longitude: $9^{\circ} 48.648^{\prime} \mathrm{E}$ et d'altitude: $15 \mathrm{~m}$. Les graines de G. $\max$ semées sont de la variété IRAT 278 de l'IRAD de Foumbot. Les insectes sur l'ensemble du site d'étude, provenaient des colonies de A. m. adansonii (variant de deux à trois entre mai 2013 et octobre 2014) et d'autres populations d'arthropodes présents dans l'environnement. La végétation était représentée par d'autres plantes cultivées puis quelques espèces naturelles de la forêt équatoriale.

\section{Méthodes}

\section{Préparation du champ expérimental, semis et entretien de la culture}

Du 14 au 19 mai 2013, puis du 21 au 22 avril 2014, les parcelles expérimentales ont été défrichées, labourées et 18 parcelles de 3 $\mathrm{m}$ de longueur, $1 \mathrm{~m}$ de largeur et $10 \mathrm{~cm}$ de hauteur ont été formés. Le 20 mai 2013 et le 25 avril 2014, le semi a été fait en ligne, à raison de deux lignes par parcelle et trois graines par poquet. Les espacements sur les lignes et entre les lignes étaient de $12,5 \mathrm{~cm}$. De la germination (survenue le 27 mai 2013 et le 30 avril 2014) à l'apparition des premières fleurs (22 juin 2013 et le 31 mai 2014), le sarclage a été régulièrement effectué à la houe, toutes les deux semaines. De la floraison à la maturation des gousses, le désherbage a été fait à la main.

\section{Détermination du système de reproduction}

Le 25 juin 2013, 12 parcelles de $G$. max porteuses de 70 plantes chacune ont été étiquetées et deux traitements constitués: traitement 1: six parcelles, porteuses de 420 plantes, non protégées; traitement 2: six parcelles, porteuses de 420 plantes protégées des insectes à l'aide des cages grillagées (longueur 3,5 m; largeur 1,5 m; hauteur 1,5 m; à mailles de $1 \mathrm{~mm}^{2}$ ) (Figure 1). Le 05 juin 2014, cette opération a été répétée. A la récolte, le nombre de gousses formées a été compté dans chacun des traitements 1 et 2 . Pour chaque traitement, l'indice de fructification $\left(\mathrm{I}_{\mathrm{fr}}\right)$ a été calculé à l'aide de la formule ci-après: $I_{f r}=F_{2} / F_{1}$, où $F_{2}$ est le nombre de gousses formées et $\mathrm{F}_{1}$ le nombre de fleurs viables initialement portées 
(Tchuenguem et al., 2001). Pour chaque saison d'observation, la différence entre les indices de fructification des deux traitements a permis de calculer les taux d'allogamie (TC) et d'autogamie (TA) au sens large, selon les formules ci-après (Tchuenguem et al., 2001): $\mathrm{TC}=\left\{\left[\left(\mathrm{I}_{\mathrm{fr} 1}-\mathrm{I}_{\mathrm{fr} 2}\right) / \mathrm{I}_{\mathrm{fr} 1}\right] * 100\right\}$ où $\mathrm{I}_{\mathrm{fr} 1}$ et $\mathrm{I}_{\mathrm{fr} 2}$ sont respectivement les indices de fructification dans le traitement libre et dans le traitement protégé; $\mathrm{TA}=(100-\mathrm{TC})$.

\section{Etude de l'activité des insectes sur les fleurs}

$\mathrm{Au}$ niveau des fleurs du traitement 1, les observations ont été effectuées tous les jours, du 2 au 20 juillet 2013 puis du 6 au 24 juin 2014, selon six tranches horaires journalières: $6-7 \mathrm{~h}, 8-9 \mathrm{~h}, 10-11 \mathrm{~h}, 12-$ $13 \mathrm{~h}, 14-15$ h et $16-17 \mathrm{~h}$. Nous passions deux à trois fois sur chaque parcelle du traitement 1 et ceci pendant chacune des tranches horaires ci-dessus. A chaque passage, les différents insectes rencontrés sur les fleurs étaient identifiés et comptés. Les insectes n'ayant pas été marqués, les résultats cumulés ont été exprimés par le nombre de visites (Tchuenguem, 2005). Les données obtenues ont permis de déterminer la fréquence de chaque espèce d'insecte $\left(F_{x}\right)$ sur les fleurs de G. max. Pour chaque saison d'étude, $\mathrm{F}_{\mathrm{x}}=\left\{\left[\mathrm{V}_{\mathrm{x}}\right.\right.$ $\left./ \mathrm{V}_{\mathrm{y}}\right]_{* 100}$, avec $\mathrm{V}_{\mathrm{x}}=$ nombre de visites de l'insecte $\mathrm{x}$ sur les fleurs du traitement aux plantes en libre pollinisation et $\mathrm{V}_{\mathrm{y}}$ le nombre de visites de tous les insectes sur ces mêmes fleurs (Tchuenguem et al., 2001).

Les produits floraux (nectar et/ou pollen) prélevés par les différents insectes ont été notés pendant les mêmes dates et tranches horaires que pour la fréquence des visites. Un insecte qui enfonce sa trompe ou sa tête dans une fleur est un chercheur de nectar; si à l'aide de ses pattes et de ses mandibules, il gratte les anthères, il s'agit d'un chercheur de pollen (Tchuenguem et al., 2001).

Les abondances de divers insectes par 1000 fleurs ont été évalués, les individus de chaque espèce d'insecte ont été comptés sur un nombre connu de fleurs épanouies; l'abondance par 1000 fleurs $\left(\mathrm{A}_{1000}\right)$ a ensuite été calculée à l'aide de la formule suivante: $\mathrm{A}_{1000}=\left[\left(\mathrm{A}_{\mathrm{x}} / \mathrm{F}_{\mathrm{x}}\right) * 1000\right]$, où $\mathrm{F}_{\mathrm{x}}$ et $\mathrm{A}_{\mathrm{x}}$ sont respectivement le nombre de fleurs et le nombre d'insectes butineurs effectivement comptés sur les fleurs laissées en libre pollinisation à l'instant $\mathrm{x}$ (Tchuenguem, 2005). Les données ont été enregistrées pendant les mêmes dates et périodes journalières que pour la fréquence des visites.

La durée des visites des différentes espèces d'insectes par fleur a été enregistrée: elle correspond au temps que met l'insecte pour prélever un produit (pollen ou nectar) sur une fleur (Tchuenguem, 2005). Elle a été enregistrée aux mêmes dates que la fréquence des visites, pendant les tranches horaires journalières suivantes : $7-8 \mathrm{~h}, 9-10 \mathrm{~h}, 11$ $12 \mathrm{~h}, 13-14 \mathrm{~h}, 15-16 \mathrm{~h}$ et $17-18 \mathrm{~h}$ à l'aide d'un chronomètre.

Pour un insecte donné, la vitesse de butinage selon Jacob-Remacle (Tchuenguem et al., 2001) correspond au nombre de fleurs visitées par minute. Ce paramètre a été enregistré pendant les mêmes tranches horaires que la durée des visites, à l'aide d'un chronomètre.

L'influence de la faune et de la flore avoisinante a été systématiquement notée lors du chronométrage de la durée des visites par fleur.

\section{Estimation de la valeur apicole}

Comme pour d'autres plantes (Tchuenguem, 2005; Djonwangwé et al., 2011; Kengni et al., 2015), la valeur apicole de G. $\max$ a été évaluée à l'aide principalement des données sur son intensité de floraison et l'attractivité de ses produits floraux vis-à-vis des ouvrières de $A . m$. adansonii.

Evaluation de l'impact des insectes anthophiles sur les rendements

Pour chaque année d'investigation, elle est basée sur: l'impact des insectes 
anthophiles sur la pollinisation, l'effet de la pollinisation sur la fructification de G. $\max$ et la comparaison des rendements fruitiers et grainiers (taux de fructification, nombre moyen de graines par gousse et pourcentage de graines normales) des traitements 1 et 2 . Le taux de fructification dû à l'influence des insectes floricoles $\left(\mathrm{P}_{\mathrm{i}}\right)$ est calculé à l'aide de la formule ci-après (Tchuenguem et al., 2001): $\mathrm{P}_{\mathrm{i}}$ $=\left\{\left[\left(\mathrm{F}_{1}-\mathrm{F}_{2}\right) / \mathrm{F}_{1}\right] * 100\right\}$ où $\mathrm{F}_{1}$ et $\mathrm{F}_{2}$ sont les taux de fructification dans les traitements 1 et 2 respectivement. Pour un traitement, le taux de fructification $=($ nombre de gousses $/$ nombre de fleurs)*100. Le pourcentage $\left(\mathrm{P}_{\mathrm{g}}\right)$ du nombre de graines par gousse attribuable à l'influence des insectes floricoles est calculé à l'aide de la formule ci-après (Tchuenguem et al., 2001): $\mathrm{P}_{\mathrm{g}}=\left\{\left[\left(\mathrm{g}_{1}-\mathrm{g}_{2}\right) / \mathrm{g}_{1}\right]_{* 100}\right\}$ où $\mathrm{g}_{1}$ et $\mathrm{g}_{2}$ sont les nombres moyens de graines par gousse dans les traitements 1 et 2 respectivement. Le pourcentage $\left(\mathrm{P}_{\mathrm{n}}\right)$ de graines normales attribuable à l'influence des insectes floricoles est calculé à l'aide de la formule ci-après (Tchuenguem et al., 2001): $P_{n}=\left\{\left(\left[P_{n 1}-\right.\right.\right.$ $\left.\left.\mathrm{P}_{\mathrm{n} 2} / / \mathrm{P}_{\mathrm{n} 1}\right] * 100\right\}$ où $\mathrm{P}_{\mathrm{n} 1}$ et $\mathrm{P}_{\mathrm{n} 2}$ sont les pourcentages de graines normales dans les traitements 1 et 2 respectivement.

\section{Analyse des données}

Le traitement des données a été fait à l'aide de la statistique descriptive (calcul des moyennes, écart-types et pourcentages), du Coefficient de corrélation $(r)$ pour l'étude des relations entre deux variables, du test $t$ de Student pour la comparaison des moyennes de deux échantillons, de l'Analyse des variances (ANOVA) pour la comparaison des moyennes de plus de deux échantillons, du Khi-carré $(\chi 2)$ pour la comparaison des pourcentages, et du logiciel Excel 2013.

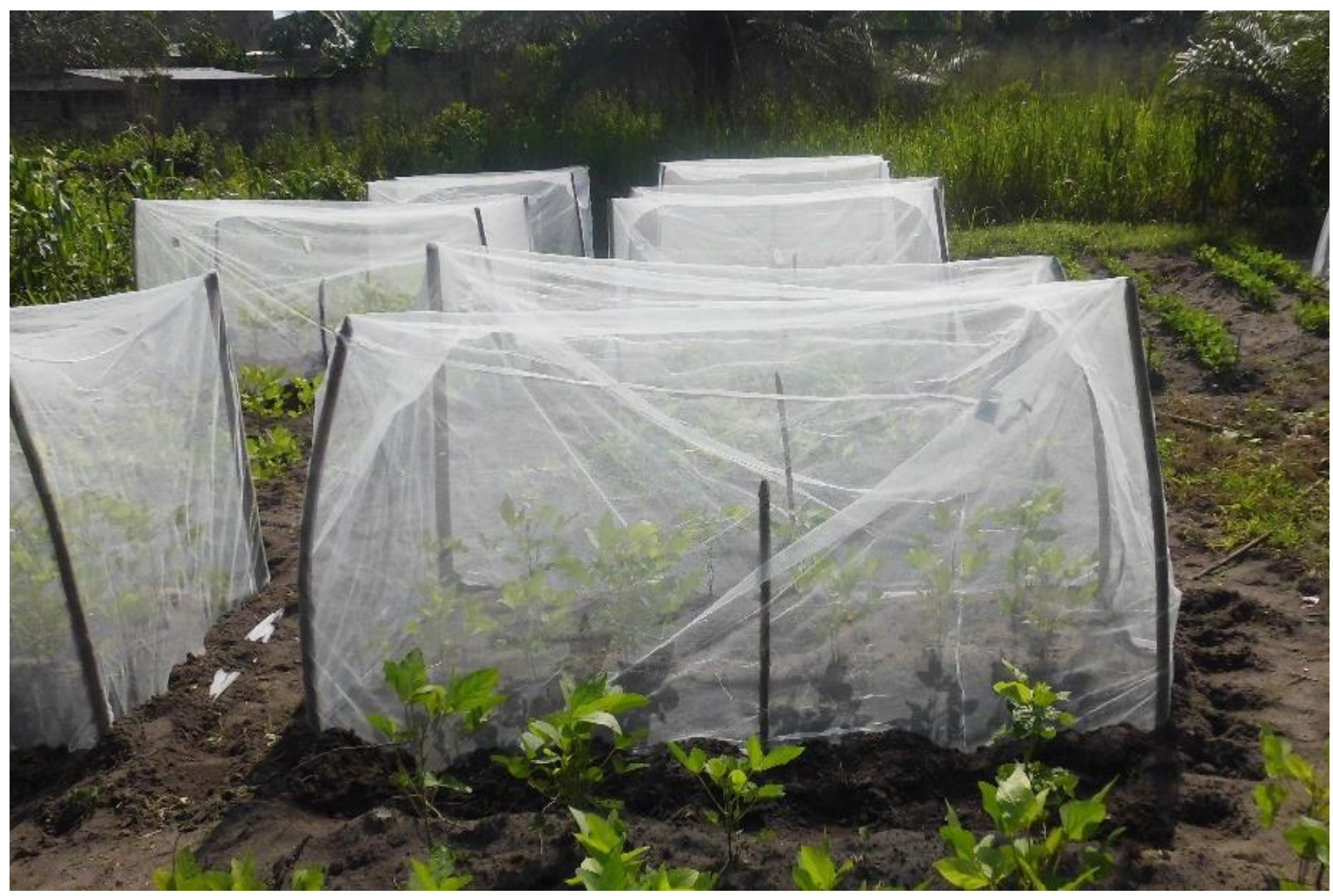

Figure 1: Vue partielle de la parcelle expérimentale de Glycine Max. 


\section{RESULTATS}

\section{Système de reproduction}

En 2013, l'indice de fructification a été de $0,62(\mathrm{~s}=0,01)$ et $0,39(\mathrm{~s}=0,03)$ pour les traitements 1 et 2 . Respectivement en 2014, les chiffres correspondants ont été de 0,55 ( $\mathrm{s}=$ $0,02)$ et $0,32(\mathrm{~s}=0,05)$. Ainsi, en $2013 T C=$ $37,09 \%$ et $T A=62,91 \%$, tandis qu'en 2014 , $T C=41,81 \%$ et $T A=58,19 \%$. Pour les deux années d'étude cumulées, $T C=39,45 \%$ et $T A$ $=60,55 \%$. Ainsi, la variété de $G$. $\max$ étudiée a un régime de reproduction mixte allogameautogame, avec prédominance de l'autogamie

\section{Diversité et fréquence des insectes floricoles de Glycine max}

En 2013 et 2014 respectivement, 1831 et 3517 visites de 13 et 14 espèces d'insectes appartenant à 8 familles et 5 ordres ont été dénombrées sur 34343 et 81041 fleurs de $G$. max. Le Tableau 1 présente la liste de ces insectes avec leur fréquence de visites. D'après les données de ce tableau, les Hyménoptères constituent l'ordre le plus important avec $94,34 \%$ de visites en 2013 et $94,87 \%$ en 2014. Ils sont principalement représentés par les Halictidés $(92,18 \%$ en 2013 et $87,13 \%$ en 2014), notamment Lasioglossum sp. (61,38\% en 2013 et $34,71 \%$ en 2014), Le. granulata $(30,53 \%$ en 2013 et $49,92 \%$ en 2014$)$ et Halictus sp. $(0,27 \%$ en 2013 et $2,50 \%$ en 2014). Les plus intéressants des autres Hyménoptères sont les Apidés $(1,85 \%$ en 2013 et $6,36 \%$ en 2014), particulièrement $A . m$. adansonii. Les autres ordres sont plus rarement observés. Il s'agit des Dictyoptères, des Coléoptères, des Lépidoptères et Diptères (moins de 5\% des visites chacun, en 2013 comme en 2014).

\section{Activités des insectes sur les fleurs de Glycine max \\ Produits floraux récoltés}

Au cours de chacune des deux périodes de floraison de G. max, les insectes ont récolté $\mathrm{du}$ nectar, du pollen ou les deux produits floraux (Figures 2 et 3). Sur les 2167 durées de visites enregistrées au cours des deux années d'observation, $306(14,12 \%)$ et 1861 $(85,88 \%)$ ont été consacrées respectivement à la récolte du nectar et des deux produits floraux.

Rythme des visites selon les étapes de la floraison

En général, les visites des insectes sont plus nombreuses sur les plants de G. max lorsque le nombre de fleurs épanouies qu'elles portent est plus élevé. Une corrélation positive et hautement significative a été trouvée entre le nombre de fleurs épanouies et le nombre de visites d'insectes en $2013(r=0,66$; $d d l=15$; $P<0,01)$ et une corrélation positive mais non significative entre ces deux paramètres en $2014(r=0,32 ; d d l=15 ; P>0,05)$.

\section{Rythme journalier des visites}

L'activité des insectes commence le matin vers $8 \mathrm{~h}$ avec l'épanouissement des fleurs et cesse fortement aux environs de $16 \mathrm{~h}$. La période journalière d'activité maximale des insectes se situe entre $12 \mathrm{~h}$ et $13 \mathrm{~h}$, aussi bien en 2013 qu'en 2014. Les conditions climatiques semblent quelquefois influencer l'activité de butinage des insectes sur les fleurs de G. $\max$ (Figure 4). En 2013, la corrélation s'est révélée positive et non significative entre le nombre de visites d'insectes et la température $(r=0,76 ; d d l=3$; $P>0,05)$, puis négative et non significative entre le nombre de visites d'insectes et l'humidité relative ( $r=-0,56 ; d d l=3 ; P>$ $0,05)$. En 2014, la corrélation s'est révélée positive et significative entre le nombre de visites des insectes et la température $(r=0,88$; $d d l=3 ; \quad P<0,05)$, négative et non significative entre le nombre de visites d'insectes et l'humidité relative de l'air ( $r=$ 0,$87 ; d d l=3 ; P>0,05$ ).

\section{Abondance des insectes butineurs}

Le plus grand nombre d'individus simultanément en activité sur une fleur était de 1 en $2013(\mathrm{n}=1827, \mathrm{~s}=0)$ comme en 2014 $(\mathrm{n}=3514, \mathrm{~s}=0)$. Les abondances par 1000 fleurs variaient de 11,91 $(\mathrm{n}=40, \mathrm{~s}=11,02)$ chez Le. granulata à 39,66 $(\mathrm{n}=96, \mathrm{~s}=31,51)$ chez Lasioglossum sp. en 2013 puis de 5,59 (n $=37, \mathrm{~s}=3,55$ ) chez A. m. adansonii à 29,02 $(\mathrm{n}=59, \mathrm{~s}=15,57)$ chez Le. granulata en 2014 (Tableau 2).

\section{Durée des visites par fleur}

La durée moyenne d'une visite d'insecte par fleur de G. max variait de 19,31 
$\sec (\mathrm{n}=75 ; \mathrm{s}=16,75)$ chez Le. granulata à $19,93 \mathrm{sec}(\mathrm{n}=644 ; \mathrm{s}=9,39) \quad$ chez Lasioglossum sp. en 2013 et de 2,91 sec ( $\mathrm{n}=$ $80 \mathrm{~s}=2,43)$ chez $A$. m. adansonii à 7,01 sec $(\mathrm{n}=702 ; \mathrm{s}=4,43)$ chez Lasioglossum sp. en 2014 (Tableau 3).

\section{Vitesse de butinage}

La vitesse moyenne de butinage des fleurs de G. max variait de 4,82 fleurs/min (n $=33 ; \mathrm{s}=3,45)$ chez Le. granulata à 6,01 fleurs/min $(\mathrm{n}=93 ; \mathrm{s}=1,54) \quad$ chez Lasioglossum sp. en 2013 et de 2,41 fleurs/min $(\mathrm{n}=143 ; \mathrm{s}=0,79)$ chez Lasioglossum sp. à 21,34 fleurs/min $(\mathrm{n}=32$; $\mathrm{s}=12,14$ ) chez Halictus sp. en 2014 (Tableau 4).

\section{Influence de la flore avoisinante}

Durant la période d'observation, plusieurs autres espèces végétales en fleurs à proximité de la parcelle de G. max étaient visitées pour leur nectar $(\mathrm{Ne})$ et/ou pour leur pollen (Po) par les insectes butineurs de cette Fabacée. Le Tableau 5 présente quelques-unes de ces plantes, ainsi que leurs insectes anthophiles. Aucun passage d'insectes des fleurs de G. max aux fleurs d'autres espèces végétales n'a été observé et vice versa. Les insectes étaient ainsi fidèles aux fleurs de $G$. max lors des voyages de butinage.

\section{Valeur apicole de $G$. $\max$}

Pendant les périodes de floraison de $G$. max, une très faible activité des ouvrières de A. m. adansonii a été notée au niveau des fleurs de cette Fabaceae: seulement 29 visites $(1,58 \%)$ consacrées à la récolte exclusive du nectar en 2013 contre 136 visites (3,86\%) en 2014; ces données mettent en évidence la non attractivité du pollen et la faible attractivité du nectar de G. max vis-à-vis de A. m. adansonii. Elles permettent de classer cette espèce végétale parmi les plantes apicoles faiblement nectarifères.

\section{Impact des insectes floricoles sur la pollinisation et les rendements de G. max}

Pendant la récolte du pollen et du nectar, la fréquence des visites avec contact entre les insectes et le stigmate étaient de 100\% aussi bien en 2013 qu'en 2014. Ces arthropodes augmentent fortement les possibilités de pollinisation de G. $\max$.

En 2013, le taux de fructification a été de $62,13 \%$ dans le traitement 1 et de $38,57 \%$ dans le traitement 2; en 2014, les chiffres correspondants étaient de $54,82 \%$ et $31,85 \%$ (Tableau 6), globalement la différence entre ces quatre pourcentages est très hautement significative $(\chi 2=2394,15 ; d d l=3$; THS). La différence entre les taux de fructification est très hautement significative en $2103\left(\chi^{2}=\right.$ $726,25 ; d d l=1$; THS $)$ comme en 2014 ( $\chi^{2}=$ 2111,$62 ; d d l=1$; THS). Pour l'ensemble des deux années d'études, le taux de fructification dû à l'influence des insectes floricoles $\left(P_{i}\right)$ a été de: $P_{i}=\left\{\left[\left(P_{i}\right.\right.\right.$ pour 2013$)+\left(P_{i}\right.$ pour 2014)] $/ 2\}=39,91 \%$.

En 2013, le nombre moyen de graines par gousse a été de 2,82 dans le traitement 1 et de 2,44 dans le traitement 2. En 2014, les chiffres correspondants étaient de 2,46 et 2,15 (Tableau 6); dans l'ensemble, la différence entre ces quatre moyennes est très hautement significative $\left(F=332,22 ; d d l_{1}=6 ; d d l_{2}=\right.$ 23424;THS). La différence entre le nombre moyen de graines par gousse est très hautement significative aussi bien en 2013 ( $t=$ 1317,95; $d d l=6789$; THS) qu'en $2014(t=$ 2612,73; $d d l=16638$; THS). Pour l'ensemble des deux années d'études, le nombre moyen de graines par gousse dû à l'influence des insectes floricoles a été de: $P_{g}=\left\{\left[\left(P_{g}\right.\right.\right.$ pour $2013)+\left(P_{g}\right.$ pour 2014) $\left.] / 2\right\}=13,04 \%$. Le nombre de graines par gousse en 2013 a été plus élevé qu'en 2014 (Tableau 6).

En 2013, le pourcentage de graines normales a été de $86,77 \%$ dans le traitement 1 et de 63,46\% dans le traitement 2. En 2014, les chiffres correspondants étaient de 95,07\% et $56,50 \%$; globalement la différence entre ces quatre pourcentages est très hautement significative ( $\chi^{2}=4730,16 ; d d l=3$; THS). La différence entre les pourcentages de graines normales est très hautement significative en 2013 ( $\chi 2=1774,49 ; d d l=1$; THS) comme en $2014(\chi 2=2727,65 ; d d l=1$; THS $)$. Pour l'ensemble des deux années d'études, le pourcentage $\left(P_{n g}\right)$ du taux de fructification dû à l'influence des insectes floricoles a été de: $P_{n g}=\left\{\left[\left(P_{n g}\right.\right.\right.$ pour 2013) $+\left(P_{n g}\right.$ pour 2014) $\left.] / 2\right\}$ $=30,72 \%$. 
Tableau 1: Insectes recensés sur les fleurs de Glycine max en juin-juillet 2013 et mai-juin 2014 à Yassa, nombre et pourcentage de visites des différents insectes.

\begin{tabular}{|c|c|c|c|c|c|c|c|c|}
\hline \multirow[b]{2}{*}{ Ordre } & \multirow[b]{2}{*}{ Famille } & \multirow{2}{*}{$\begin{array}{c}\text { Insectes } \\
\begin{array}{c}\text { Genre, espèce, sous- } \\
\text { espèce }\end{array}\end{array}$} & \multicolumn{2}{|c|}{2013} & \multicolumn{2}{|c|}{2014} & \multicolumn{2}{|c|}{ Total $_{2013 / 2014}$} \\
\hline & & & $\mathrm{n}_{1}$ & $p_{1}(\%)$ & $\mathbf{n}_{2}$ & $p_{2}(\%)$ & $\mathbf{n}_{\mathrm{T}}$ & $\mathrm{p}_{\mathrm{T}}(\%)$ \\
\hline Dictyoptera & Mantidae & Mantis religiosa & 4 & 0,21 & 3 & 0,08 & 7 & 0,13 \\
\hline \multirow[t]{4}{*}{ Diptera } & Muscidae & Musca domestica & 38 & 2,07 & 28 & 0,79 & 64 & 1,19 \\
\hline & Syrphidae & (sp.1) & 4 & 0,21 & 20 & 0,56 & 24 & 0,44 \\
\hline & & (sp.2) & 7 & 0,18 & 35 & 0,99 & 42 & 0,78 \\
\hline & & Total Syrphidae & 11 & 0,39 & 55 & 1,55 & 66 & 1,22 \\
\hline \multirow[t]{3}{*}{ Coleoptera } & Chrysomelidae & (1 sp.) & - & - & 48 & 1,36 & 48 & 0,49 \\
\hline & Meleonidae & Lagria villosa & 29 & 1,58 & - & - & 29 & 0,54 \\
\hline & Total Coleoptera & & 29 & 1,58 & 48 & 1,36 & 77 & 1,03 \\
\hline Lepidoptera & Pieridae & Eurema sp. & 17 & 0,38 & 28 & 0,79 & 45 & 0,84 \\
\hline \multirow[t]{11}{*}{ Hymenoptera } & Apidae & $\begin{array}{c}\text { Apis mellifera } \\
\text { adansonii }\end{array}$ & 29 & 1,58 & 136 & 3,86 & 165 & 3,08 \\
\hline & Halictidae & Halictus sp. & 5 & 0,27 & 88 & 2,5 & 93 & 1,73 \\
\hline & & Lasioglossum sp. & 1124 & 61,38 & 1221 & 34,71 & 2345 & 43,84 \\
\hline & & Leuconomia granulata & 559 & 30,53 & 1756 & 49,92 & 2315 & 43,28 \\
\hline & & Total Halictidae & 1688 & 92,18 & 3065 & 87,13 & 4753 & 88,85 \\
\hline & Megachilidae & Chalicodoma sp. & 4 & 0,21 & 17 & 0,48 & 21 & 0,39 \\
\hline & & Megachile sp. 1 & 8 & 0,42 & 60 & 1,70 & 68 & 1,27 \\
\hline & & Megachile sp. 2 & - & - & 45 & 1,27 & 49 & 0,91 \\
\hline & & Megachile sp. 3 & 3 & 0,16 & 32 & 0,91 & 35 & 0,65 \\
\hline & & Total Megachilidae & 15 & 0,79 & 154 & 4,36 & 173 & 3,22 \\
\hline & Total & Hymenoptera & 1728 & 94,34 & 3338 & 94,87 & 5070 & 94,76 \\
\hline Total & & & 1831 & 100 & 3517 & 100 & 5348 & 100 \\
\hline \multicolumn{3}{|c|}{ Nombre d'espèces } & \multicolumn{2}{|c|}{13 espèces } & \multicolumn{2}{|c|}{14 espèces } & \multicolumn{2}{|c|}{15 espèces } \\
\hline
\end{tabular}

$\mathrm{n}_{1}$ : nombre de visites sur 34343 fleurs en 17 jours; $\mathrm{n}_{2}$ : nombre de visites sur 81041 fleurs en 17 jours; $\mathrm{n}_{\mathrm{s}}$ : nombre total de visites pour les deux années; $\mathrm{p}_{1}, \mathrm{p}_{2}, \mathrm{p}_{\mathrm{T}}$ : pourcentage de visites; $\mathrm{p}_{1}=\left(\mathrm{n}_{1} / 1831\right) * 100 ; \mathrm{p}_{2}=\left(\mathrm{n}_{2} / 3517\right)$ $* 100 ; \mathrm{p}_{\mathrm{T}}=\left(\mathrm{n}_{\mathrm{T}} / 5348\right) * 100$.

Comparaison des pourcentages de visites 2013/2014: Apis mellifera adansonii : $\chi^{2}=20,99$ (ddl $=1 ; P<0,001$; THS); Halictus sp. : $\chi^{2}=35,01$ (ddl $=1 ; P<0,001$; THS); Leuconomia granulata : $\chi^{2}=184,58$ (ddl $=1 ; P<0,001$; THS); Lasioglossum sp. : $\chi^{2}=347,86$ ( $(d d l=1 ; P<0,001 ;$ THS). 


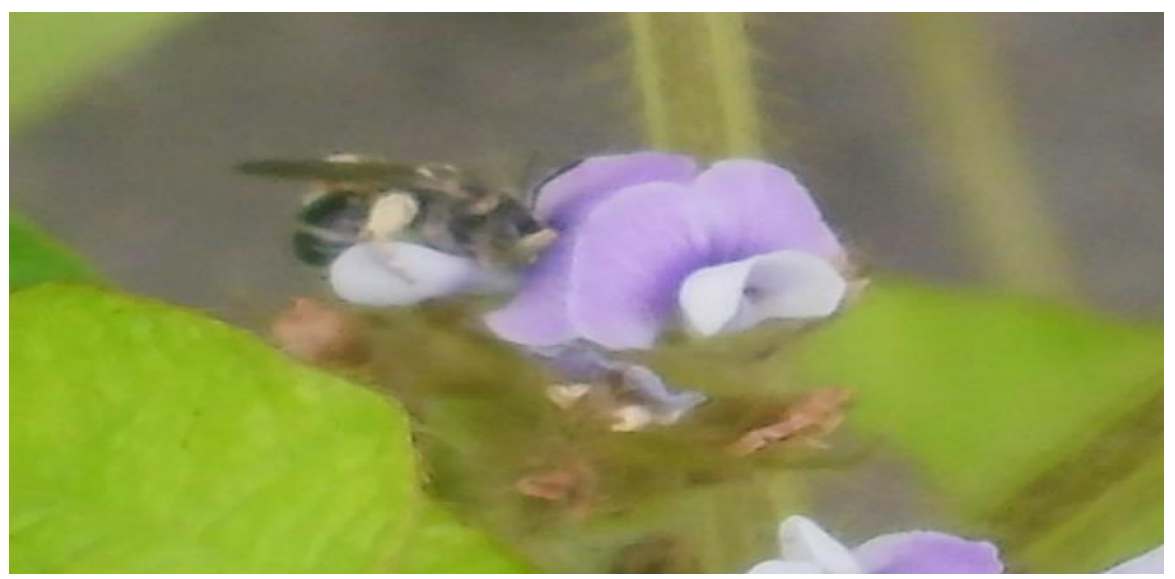

Figure 2: Lasioglossum sp. recoltant le nectar dans une fleur de Glycine max.

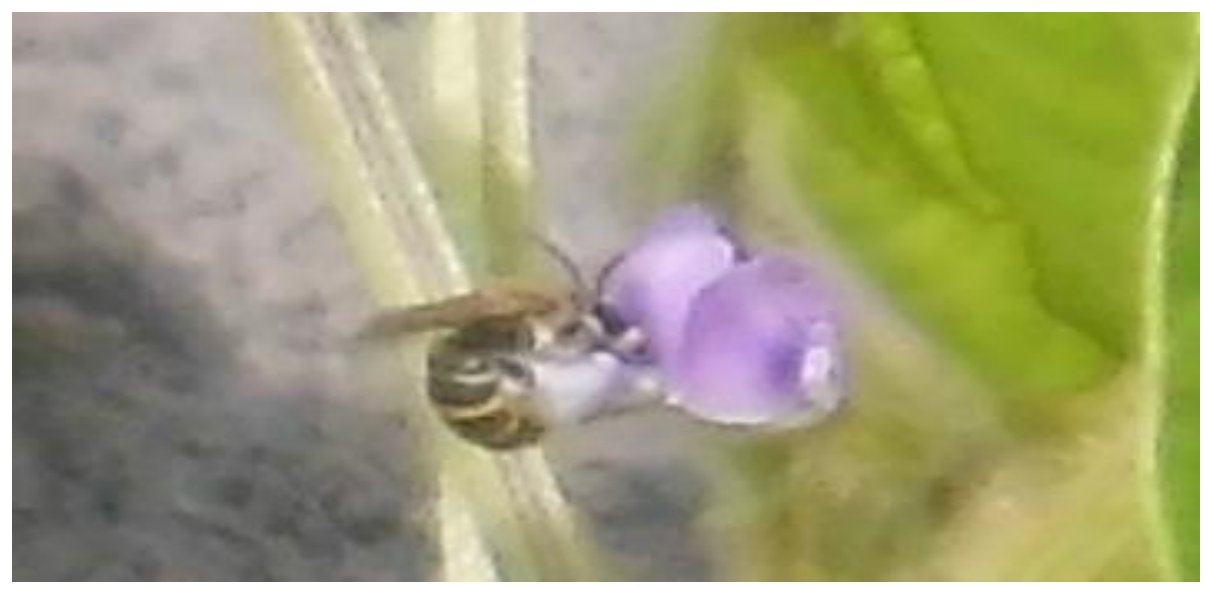

Figure 3: Leuconomia granulata recoltant le nectar dans une fleur de Glycine max.

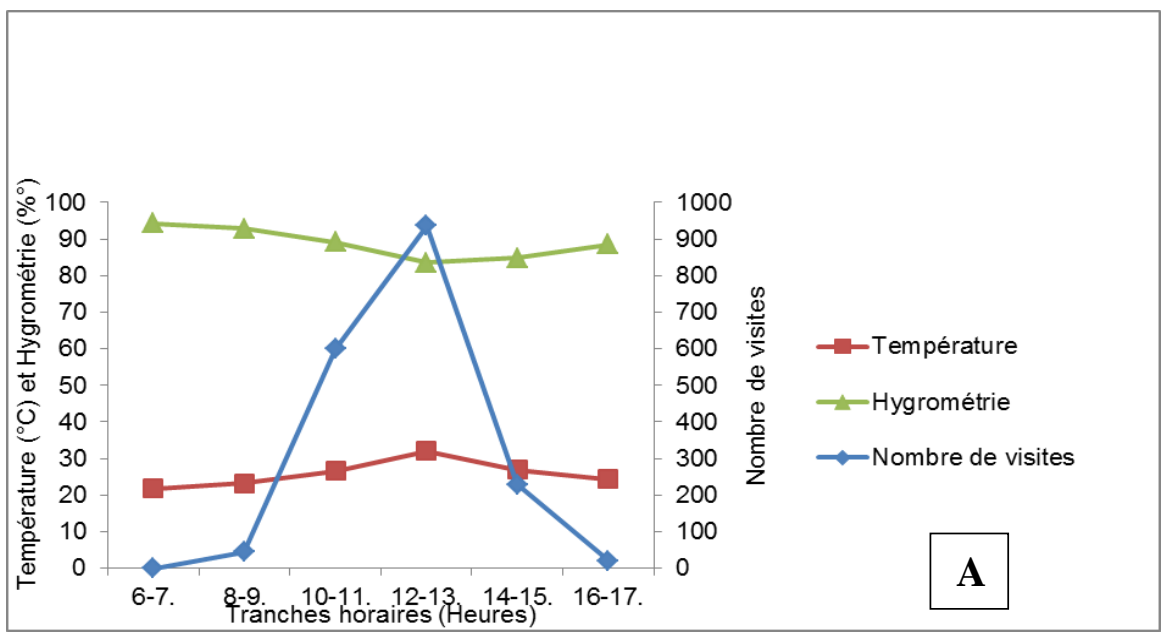




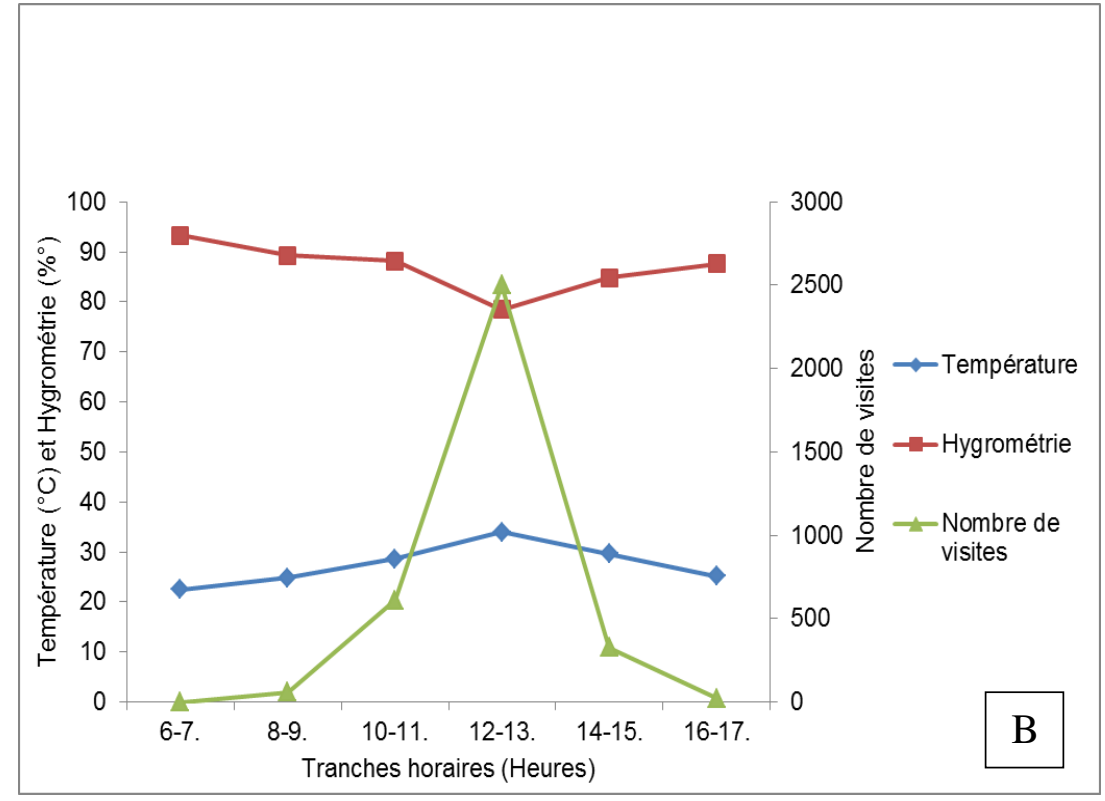

Figure 4: Distribution du nombre de visites d'insectes sur les fleurs de Glycine max, puis de la température et de l'hygrométrie de l'air selon les tranches horaires journalières en 2013 (A) et 2014 (B) à Yassa.

Tableau 2: Abondance de quelques insectes par 1000 fleurs de Glycine max en 2013 et 2014 à Yassa.

\begin{tabular}{lllllll}
\hline Insectes & Années & $\mathbf{n}$ & \multicolumn{5}{c}{ Abondance par 1000 fleurs } \\
\cline { 5 - 7 } & & & $\boldsymbol{m}$ & $\boldsymbol{s}$ & mini & maxi \\
\hline A.m. adansonii & 2013 & - & - & - & - & - \\
& 2014 & 37 & 5,59 & 3,55 & 1,26 & 18,58 \\
Halictus sp. & 2013 & - & - & - & - & - \\
& 2014 & 51 & 22,32 & 12,72 & 36 & 65,11 \\
Lasioglossum sp. & 2013 & 96 & 39,66 & 31,51 & 8,21 & 176,47 \\
& 2014 & 90 & 25,95 & 12,77 & 1,80 & 44,60 \\
Le. Granulata & 2013 & 40 & 11,91 & 11,02 & 2,63 & 56,17 \\
& 2014 & 59 & 29,02 & 15,57 & 4,70 & 93,75 \\
\hline
\end{tabular}

Comparaison des moyennes: $F=16,95\left(d d l_{1}=6 ; d d l_{2}=393 ; P<0,001\right.$; THS);

Lasioglossum sp.: $t=26,04$ ( $d d l=184 ; P<0,001$; THS);

Le. granulata $: t=29,02(d d l=97 ; P<0,001 ;$ THS $)$. 
Tableau 3 : Durée des visites de quelques insectes sur les fleurs de Glycine max en 2013 et 2014 à Yassa.

\begin{tabular}{|c|c|c|c|c|c|c|}
\hline \multirow[t]{2}{*}{ Insectes } & \multirow[t]{2}{*}{ Années } & \multirow[t]{2}{*}{$\mathbf{n}$} & \multicolumn{4}{|c|}{ Durée d'une visite par fleur (seconde) } \\
\hline & & & $\boldsymbol{M}$ & $s$ & $\operatorname{mini}$ & $\operatorname{maxi}$ \\
\hline \multirow[t]{2}{*}{ A.m. adansonii } & 2013 & - & - & - & - & - \\
\hline & 2014 & 80 & 2,91 & 2,43 & 1 & 19 \\
\hline \multirow[t]{2}{*}{ Halictus sp. } & 2013 & - & - & - & - & - \\
\hline & 2014 & 226 & 4,66 & 4,30 & 1 & 38 \\
\hline \multirow[t]{2}{*}{ Lasioglossum sp. } & 2013 & 644 & 19,93 & 9,39 & 2 & 45 \\
\hline & 2014 & 702 & 7,01 & 4,43 & 1 & 26 \\
\hline \multirow[t]{2}{*}{ Le. Granulata } & 2013 & 75 & 19,31 & 16,75 & 4 & 53 \\
\hline & 2014 & 440 & 4,92 & 4,05 & 1 & 43 \\
\hline
\end{tabular}

Tableau 4 : Vitesse de butinage de quelques insectes sur les fleurs de Glycine max en 2013 et 2014 à Yassa.

\begin{tabular}{|c|c|c|c|c|c|c|}
\hline \multirow[t]{2}{*}{ Insectes } & \multirow[t]{2}{*}{ Années } & \multirow[t]{2}{*}{$\mathbf{n}$} & \multicolumn{4}{|c|}{ Vitesse de butinage fleurs/(minute) } \\
\hline & & & $M$ & $s$ & mini & maxi \\
\hline \multirow[t]{2}{*}{ A.m. adansonii } & 2013 & - & - & - & - & - \\
\hline & 2014 & 81 & 16,64 & 6,95 & 7,92 & 40 \\
\hline \multirow[t]{2}{*}{ Halictus sp. } & 2013 & - & - & - & - & - \\
\hline & 2014 & 32 & 21,34 & 12,14 & 36 & 64 \\
\hline \multirow[t]{2}{*}{ Lasioglossum sp. } & 2013 & 93 & 6,01 & 1,54 & 1,54 & 10,58 \\
\hline & 2014 & 143 & 2,41 & 0,79 & 1,18 & 5 \\
\hline \multirow[t]{2}{*}{ Le. Granulata } & 2013 & 33 & 4,82 & 3,45 & 0,89 & 18 \\
\hline & 2014 & 91 & 3,93 & 2,59 & 1,23 & 18 \\
\hline
\end{tabular}


Tableau 5: Aliments récoltés par quelques insectes sur les fleurs de quelques espèces de plantes avoisinant Glycine max en 2013 et 2014 à Yassa.

\begin{tabular}{lcccccc}
\hline \multirow{2}{*}{ Espèces végétales } & \multicolumn{2}{c}{ Apis mellifera adansonii } & \multicolumn{2}{c}{ Leucomia granulata } & \multicolumn{2}{c}{ Lasioglossum sp. } \\
\cline { 2 - 6 } & $\mathrm{Ne}$ & Po & $\mathrm{Ne}$ & Po & Ne & Po \\
\hline Bidens pilosa & $+(\mathrm{A}, \mathrm{B})$ & $+(\mathrm{A}, \mathrm{B})$ & $+(\mathrm{A}, \mathrm{B})$ & $+(\mathrm{A}, \mathrm{B})$ & $+(\mathrm{A}, \mathrm{B})$ & $+(\mathrm{A}, \mathrm{B})$ \\
Brachiara brizantha & & $++(\mathrm{A}, \mathrm{B})$ & & $+(\mathrm{A}, \mathrm{B})$ & & $+(\mathrm{B})$ \\
Mimosa pudica & & $++(\mathrm{A}, \mathrm{B})$ & & $++(\mathrm{A})$ & \\
Arachis hypogaea & & & $+(\mathrm{A}, \mathrm{B})$ & $++(\mathrm{A}, \mathrm{B})$ \\
Zea mayis & & $++(\mathrm{A}, \mathrm{B})$ & & $+(\mathrm{A}, \mathrm{B})$ & $+(\mathrm{A}, \mathrm{B})$ \\
Psidium guajava & & $++(\mathrm{A}, \mathrm{B})$ & & $+(\mathrm{A}, \mathrm{B})$ & $+(\mathrm{A}, \mathrm{B})$ \\
Mimosa invisa & & $++(\mathrm{A}, \mathrm{B})$ & & $++(\mathrm{A}, \mathrm{B})$ & & $++(\mathrm{A}, \mathrm{B})$ \\
Senna mimosoides & $++(\mathrm{A}, \mathrm{B})$ & $++(\mathrm{A}, \mathrm{B})$ & & & & \\
Manihot esculenta & $+(\mathrm{A}, \mathrm{B})$ & $+(\mathrm{A}, \mathrm{B})$ & $+(\mathrm{A}, \mathrm{B})$ & $+(\mathrm{A}, \mathrm{B})$ & & \\
Oxalis barrelieri & & & $++(\mathrm{A}, \mathrm{B})$ & & $+(\mathrm{A}, \mathrm{B})$ & $+(\mathrm{A}, \mathrm{B})$ \\
\hline
\end{tabular}

+ : faible récolte ; ++ : forte récolte ; A : 2013 ; B : 2014 ; Ne : Nectar ; Po : Pollen.

Tableau 6 : Taux de fructification, nombre moyen de graines par gousse et pourcentage des graines normales selon les traitements de Glycine max en 2013 et 2014 à Yassa.

\begin{tabular}{lcccccccccc}
\hline Traitements & Années & NFE & NFF & $\begin{array}{c}\text { TF } \\
(\boldsymbol{\%})\end{array}$ & \multicolumn{2}{c}{ Graines/gousse } & NTG & NGN & \% GN \\
& & & & & $\boldsymbol{n}$ & $\boldsymbol{m}$ & $\boldsymbol{s}$ & & \\
\hline & & & & & & & & \\
\hline $1(\mathrm{Fl})$ & 2013 & 7223 & 4488 & 62,13 & 4488 & 2,82 & 0,41 & 13087 & 11356 & 86,77 \\
$2(\mathrm{Fi})$ & & 5970 & 2303 & 38,57 & 2303 & 2,44 & 0,49 & 8075 & 5125 & 63,46 \\
$1(\mathrm{Fl})$ & 2014 & 18785 & 10298 & 54,82 & 10298 & 2,46 & 0,51 & 40272 & 38287 & 95,07 \\
$2(\mathrm{Fi})$ & & 19999 & 6342 & 31,85 & 6342 & 2,15 & 0,38 & 15957 & 9017 & 56,50 \\
\hline
\end{tabular}

NFE: nombre de fleurs étudiées; NFF: nombre de fruits formés; TF: taux de fructification; NTG: nombre total de graines; NGN: nombre de graines normales; \%GN: pourcentage de graines normales; Fl: plantes en libre pollinisation; Fi: plantes protégées des insectes.

Comparaison des taux de fructification: $\chi^{2}=2394,15$ (ddl $=3 ; P<0,001$; THS);

$\mathrm{F}_{1} 2013 / \mathrm{F}_{\mathrm{i}} 2013: \chi^{2}=726,25$ (ddl = 1; $\left.P<0,001 ; \mathrm{THS}\right) ; \mathrm{F}_{1} 2014 / \mathrm{F}_{\mathrm{i}} 2014: \chi^{2}=2111,62$ (ddl = 1; $P<0,001$; THS); $\mathrm{F}_{1} 2013 / \mathrm{F}_{1} 2014: \chi 2=113,79(d d l=1 ; P<0,001 ; \mathrm{THS})$;

Nombres moyens de graines/gousse: $F=332,22\left(d d l_{l}=6\right.$; $d d_{2}=23424 ; P<0,001$; THS $)$;

$\mathrm{F}_{1} 2013 / \mathrm{F}_{\mathrm{i}} 2013: t=1387,11(d d l=6789 ; P<0,001 ;$ THS $) ; \mathrm{F}_{1} 2014 / \mathrm{F}_{\mathrm{i}} 2014: t=2612,73(d d l=16639 ; P<0,001$; THS); $\mathrm{F}_{1} 2013 / \mathrm{F}_{1} 2014: t=2335,18$ ( $d d l=14784 ; P<0,001$; THS);

Pourcentages de graines normales: $\chi^{2}=4730,16(d d l=3 ; P<0,001$; THS);

$\mathrm{F}_{1} 2013 / \mathrm{F}_{\mathrm{i}} 2013: \chi^{2}=1574,49(d d l=1 ; P<0,001 ; \mathrm{THS}) ; \mathrm{F}_{1} 2014 / \mathrm{F}_{\mathrm{i}} 2014: \chi 2=12727,65$ (ddl = 1; $P<0,001 ;$ THS $)$; $\mathrm{F}_{1} 2013 / \mathrm{F}_{2} 2014: \chi 2=1049,67(d d l=1 ; P<0,001 ; \mathrm{THS})$.

\section{DISCUSSION}

Activité des insectes sur les fleurs de Glycine max, valeur apicole et rendement de la Fabaceae

A Yassa (Douala, Cameroun), les principaux insectes anthophiles de G. max appartiennent à l'ordre des Hyménoptères, avec la famille des Halictidés qui est la plus abondante. Ces résultats sont similaires à ceux obtenus par Dounia (2015) qui a travaillé sur l'activité de quelques insectes anthophiles du soja à Mayel-Ibbé (Maroua) et a trouvé que l'ordre le plus important est celui des Hyménoptères $(72,46 \%$ des visites) avec la famille des Halictidés qui est la plus abondante. 
La diversité et l'abondance des insectes anthophiles de G. $\max$ peuvent varier avec les régions. Ainsi, à Maroua et à Ngaoundéré au Cameroun, contrairement aux résultats obtenus Tchuenguem et Dounia (2014); Kengni et al. (2015) ont respectivement trouvé que $A . m$. adansonii était l'insecte le plus fréquent sur les fleurs de la Fabaceae. Aux Etats Unis (Rortais et al., 2005) et au Brésil (Milfont et al., 2013) A mellifera a été trouvé comme l'insecte anthophile le plus abondant sur G. max.

Dans l'ensemble, les insectes sont abondants sur les fleurs aux environs de la mijournée avec un pic d'activité situé entre 12 et 13h. Sur la même plante à Maroua, Tchuenguem et Dounia (2014) ont trouvé que le pic d'activité des insectes était situé entre 10 et $11 \mathrm{~h}$ tandis que Kengni et al. (2015) à Ngaoundéré ont trouvé un pic entre 13 et 14h. Ce pic journalier d'activité correspond au moment de la journée où les produits floraux de G. max sont disponibles en plus grandes quantités. La corrélation positive et significative entre le nombre de fleurs épanouies et le nombre de visites d'insectes démontrent la forte attractivité exercée par les produits foraux de G. $\max$ vis-à-vis de ses insectes floricoles.

La durée moyenne d'une visite de récolte de pollen et/ou du nectar varie avec l'insecte et ceci d'une année à une autre. La différence significative observée entre la durée des visites en 2013 et 2014 semble être liée à l'accessibilité et à la disponibilité des produits floraux de G. max.

Les interruptions des visites ont lieu à la suite des pluies violentes, des collisions entre les visiteurs, des tentatives de capture du visiteur par un prédateur ou de l'approche de la fleur déjà occupée par un premier visiteur. L'interruption des visites chez les insectes peut entraîner la réduction de la durée d'une visite par fleur et la perte d'une partie du pollen transporté. Ceci a pour conséquence la prolongation de l'activité exploratrice de l'insecte lors d'un voyage de butinage.

Pour l'ensemble des insectes butineurs, le passage de G. max à une autre plante avoisinante n'a pas été observé. Ceci prouve que les butineuses des fleurs de G. max étaient fidèles à cette Fabaceae. Chez A. mellifera, ce phénomène est connu sous l'expression «constance florale» (Basualdo et al., 2000). La constance florale est un aspect important dans la gestion de la pollinisation. Au Cameroun, la fidélité de l'abeille domestique a été également signalé sur: Callistemon rigidus, Syzygium guineense var. macrocarpum et Voacanga africana (Tchuenguem, 2005); Vitellaria paradoxa (Djonwangwe et al., 2011). Chez A. m. adansonii, ladite constance florale s'explique par le fait que chez cette abeille, la butineuse est en général capable de mémoriser et de reconnaître la forme, la couleur et l'odeur de fleurs visitées lors des voyages de butinage antérieurs (Wright et al., 2002).

Une très faible activité des ouvrières de A. m. adansonii a été notée sur les fleurs de $G$. max durant les deux années d'investigation. Ceci serait dû au fait que les produits floraux de cette plante ne sont pas très attractifs pour cette abeille dans ces conditions d'observation. Elle serait aussi due au faible nombre de colonies de A. m. adansonii dans l'environnement du site d'étude. La valeur apicole de cette Fabaceae est faible à Yassa. Ce résultat n'est pas en accord avec les données obtenues à Maroua et à Ngaoundéré au Cameroun qui montrent que G. $\max$ est une plante apicole fortement nectarifère (Dounia et Tchuenguem, 2014; Kengni et al., 2015). Pour chacune des deux années d'investigation, les rendements en gousses et en graines des fleurs de G. $\max$ en libre pollinisation et butinées par les insectes ont été plus élevés que ceux des fleurs protégées.

\section{Impact de l'activité des insectes sur la pollinisation et les rendements de Glycine $\max$}

Au cours de leur activité de récolte du nectar et du pollen, les insectes rentrent fréquemment en contact avec le stigmate. Ils peuvent ainsi intervenir directement dans l'autopollinisation, en mettant le pollen d'une fleur sur le stigmate de celle-ci. Cette observation est en accord avec celles de 
Tchuenguem (2005), Tchuenguem et Dounia (2014), Kengni et al. (2015) et Dounia (2015) qui indiquent que les pollinisateurs efficaces d'une espèce végétale se recrutent parmi les insectes floricoles qui sont régulièrement des contacts avec le stigmate et les anthères des fleurs visitées. Les insectes secouent les fleurs pendant leurs visites. $\mathrm{Ce}$ mouvement faciliterait la libération du pollen pour l'occupation optimale du stigmate. La charge optimale de pollen sur le stigmate serait favorable à la formation des gousses et des graines. En effet, Tchuenguem et al. (2001) ont montré qu'une pollinisation efficace permet l'augmentation des rendements et la qualité du fruit est meilleure. En agitant les fleurs, les insectes anthophiles interviennent dans l'autopollinisation. Comme l'avait signalé Tchuenguem et Dounia (2014), le rôle des différents insectes sur l'autopollinisation du soja serait proportionnel à leur aptitude à provoquer l'agitation de la fleur. Pour cette raison, il est attribuable principalement aux Apoïdes. Ce phénomène a été également démontré par Rortaisa et al. (2005), Tchuenguem et Dounia (2014) et Kengni et al. (2015) sur G. max.

\section{Conclusion}

A Douala, G. $\max$ est une plante nectarifère et pollinifère qui bénéficie de la pollinisation par les insectes. En comparant le rendement des plantes non protégées à celui des plantes protégées des insectes, il est apparu que les insectes anthophiles augmentent significativement le taux de fructification, le nombre moyen de graines par gousse ainsi que le pourcentage de graines normales aussi bien en 2013 et qu'en 2014. La grande majorité des insectes inventoriés sur les fleurs du soja sont des pollinisateurs de cette plante. Les plus intéressants d'entre eux sont des Abeilles de la famille des Halictidés qui sont de bons récolteurs du nectar et du pollen de la Fabacée. Le traitement des plants de soja aux pesticides chimiques est à éviter pendant la période de floraison, afin de ne pas menacer les insectes pollinisateurs.

\section{CONFLIT D'INTERETS}

Les auteurs déclarent qu'il n'y a aucun conflit d'intérêts.

\section{CONTRIBUTIONS DES AUTEURS}

$\mathrm{T}$ a mené les manipulations sur le terrain et au laboratoire. Les résultats obtenus ont été analysés avec l'apport de F-NTF qui a participé activement à la rédaction de ce manuscrit.

\section{REMERCIEMENTS}

Les auteurs remercient le Laboratoire de Zoologie Appliquée de l'Université de Ngaoundéré.

\section{REFERENCES}

Basualdo M, Bedascarrasbure E, De Jong D. 2000. Africanized honey bees (Hymenoptera : Apidae) have a greater fidelity to sunflowers than European bees. Journal of Economic Entomology, 2: 304-307. DOI: http://dx.doi.org/10.1603/0022-049393.2.304.

Djonwangwe D, Tchuenguem FFN, Messi J, Brückner D. 2011. Impact de l'activité de butinage de Apis mellifera adansonii Latreille (Hymenoptera : Apidae) sur la pollinisation et la chute des jeunes fruits du karité Vitellaria paradoxa (Sapotaceae) à Ngaoundéré (Cameroun). International Journal of Biological and Chemical Sciences, 5(4): 1538-1551.

Otiobo AEN, Tchuenguem FFN, Djieto CL. 2015. Foraging and pollination behavior of Apis mellifera adansonii (Hymenoptera Apidae) on Physalis micrantha (Solanales Solanaceae) flowers at Bambui (Nord West, Cameroon). Journal of Entomology and Zoology Studies, 3(6): 250-256.

Dounia, 2015. Activités de butinage et de pollinisation de Apis mellifera adansonii 
Latreille

(Apidae, Apinae),

Macronomia vulpina (Gerstaecker, 1857) (Hymenoptera: Halictidae) et Lipotriches collaris (Vachal, 1903) (Hymenoptera: Halictidae) sur les fleurs de Gossypium hirsutum L. (Malvaceae) et Glycine max L. (Fabaceae) à Maroua (Cameroun). Thèse de Doctorat/Ph.D, Université de Ngaoundéré, Ngaoundéré, p.148.

DSCE. 2009. Document de Stratégie pour la Croissance et l'Emploi. MINEPAT: Yaoundé.

DSCE. 2010. Document de Stratégie pour la Croissance et l'Emploi. MINEPAT : Yaoundé.

Gallais N, Salles J-M, Settele J, Vaissière BE. 2009. Economic valuation of the vulnerability of world agriculture confronted with pollinator decline. Ecological Economics, 68: 810-821.

Gilles S. 2000. Vivre à Douala. L'Imaginaire et l'Action dans une Ville Africaine en Crise. Harmattan: Paris.

Kengni SB, Tchuenguem FFN, Ngakou A. 2015. Impact of the foraging activity of Apis mellifera adansonii Latreille (Hymenoptera: Apidae) and Bradyrhizobium fertilizer on pollination and yield components of Glycine max L. (Fabaceae) in the field. International Journal of Biological Research. 3(x) (2015).

Kengue J, Tchuenguem FFN, Adewusi HG. 2002. Towards the improvement of safou (Dacryodes edulis): population variation and reproductive biology. Forests Trees and Livelihoods, 12: 73-84.

Kingha TMB, Tchuenguem FFN, Brückner D. 2014. Diversité des insectes floricoles et son impact sur les rendements fruitiers et grainiers de Arachis hypogaea L. (Fabaceae) à Dang (NgaoundéréCameroun). International Journal Biological and Chemical Sciences, 8(3): 983-997.

Milfont MO, Epifania EM, Rocha, Afonso ON, Lima Freitas BM. 2013. Higher soybean production using honeybee and wild pollinators, a sustainable alternative to pesticides and autopollination. Environmental Chemistry Letters. DOI: http://dx.doi.org/10.1007/s10311-01303.

Morrison M, Vaissière B, Rodet G. 2000. La pollinisation des cultures entomophiles. Bulletin Semences, 153: 31-34.

Ngamo Tinkeu LS, Tamgno BR, Gandebe M. 2016. Bioactivity of flours of seeds of leguminous crops Pisum sativum, Phaseolus vulgaris and Glycine max used as botanical insecticides against Sitophilus oryzae Linnaeus (Coleoptera: Curculionidae) on sorghum grains. Int. J. Biol. Chem. Sci., 10(3): 919-927. DOI: http://dx.doi.org/10.4314/ijbcs.v10i3.1

Noubissié TJB, Tchuenguem FFN Tchako TL. 2012. Role of Lepidoptera as pollinators on the breeding systems of Striga hermonthica (Del.) benth under the Guinea savannah zone conditions. Annals of Biological Research, 3(6): 2821-2828.

Pando BJ, Tchuenguem FFN, Djonwangwe D, Tamesse JL. 2014. The importance of single floral visit of Chalicodoma rufipes L. (Hymenoptera: Megachilidae) in the pollination and yield of Vigna unguiculata (L.) Walp. 1843 (Fabaceae) in Cameroon. International Journal of Agronomy and Agricultural Research, 4(4): 179-187.

Raemaekers RH. 2001. Agriculture en Afrique Tropicale. De Slegte ; p. 860-882. ISBN: 9789080682221 ;

https://www.deslegte.be/agriculture-enafrique-tropicale-150637/

Rortais A, Arnold G, Halmb MP, TouffetBriens BF. 2005. Modes of honeybees exposure to systemic insecticides: estimated amounts of contaminated pollen and nectar consumed by different categories of bees. Apidologie, 36(1): 7183. DOI: http://dx.doi.org/10.1051/ apido:2004071.

Tchuenguem FFN. 2005. Activité de butinage et de pollinisation d'Apis mellifera adansonii Latreille (Hymenoptera: Apidae, Apinae) sur les fleurs de trois 
plantes à Ngaoundéré (Cameroun): Callistemon rigidus (Myrtaceae), Syzygium guineense var. macrocarpum (Myrtaceae) et Voacanga africana (Apocynaceae). Thèse de Doctorat d'Etat, Université de Yaoundé I, Yaoundé, p.103.

Tchuenguem FFN, Messi J, Pauly A. 2001. Activité de Meliponula erythra sur les fleurs de Dacryodes edulis et son impact sur la fructification. Fruits, 56(3): 179188.

Tchuenguem FFN, Messi J, Pauly A. 2002. L'activité de butinage des Apoïdes sauvages (Hymenoptera: Apoidea) sur les fleurs de maïs à Yaoundé (Cameroun) et réflexion sur la pollinisation des graminées tropicales. Biotechnol. Agron. Soc. Environ., 6(2): 87-98.

Tchuenguem FFN, Mapongmetsem PM, Hentchoya HJ, Messi J. 2004. Exploitation des fleurs de quatre plantes oléagineuses par Apis mellifera adansonii à Ngaoundéré (Cameroun): Bombax pentandrum, Vitellaria paradoxa, Lophira lanceolata et Dacryodes edulis. Procédés Biologiques et Alimentaires, 2: 27-36.
Tchuenguem FFN, Dounia 2014. Foraging and pollination behavior of Apis mellifera adansonii Latreille (Hymenoptera: Apidae) on Glycine max L. (Fabaceae) flowers at Maroua. Journal of Research in Biology, 4(1): 209-219.

Vaughton G, Ramsey M, Johnson SD. 2010. Pollination and late-acting selfin compatibility in Cyrtanthus breviflorus (Amaryllidaceae): implications for seed production. Annals of Botany, 106: 547555.

Tien HH, Hien TM, Son MT, Herridge D. 2002. Rhizobial Inoculation and $\mathrm{N}_{2}$ fixation of soybean and mungbean in the eastern region of South Vietnam. In Inoculants and Nitrogen Fixation of Legumes in Vietnam, Herridge D (ed). ACIAR Proceedings 109e.

Wright GA, Skinner BD, Smith BH. 2002. Ability of honeybee, Apis mellifera, to detect and discriminate odors of varieties of canola (Brassica rapa and Brassica napus) and snopgragon flowers (Antirrhinum majus). Journal of Chemical Ecology, 28(4): 721-740. 